\title{
A New UTD for the Electromagnetic Plane Wave Scattering by a Canonical Circular Cylinder with an Impedance Boundary Condition
}

\author{
Andres G. Aguilar and Prabhakar H. Pathak
}

\begin{abstract}
A UTD solution is developed for describing the scattering by a circular cylinder with an impedance boundary condition (IBC), when it is illuminated by an obliquely incident electromagnetic ( $\mathrm{EM}$ ) plane wave. The solution to this canonical problem will be crucial for the construction of a more general UTD solution valid for an arbitrary smooth convex surface with an IBC, when it is illuminated by an arbitrary EM ray optical field. The canonical solution is uniformly valid across the surface shadow boundary that is tangent to the surface at grazing incidence. This canonical solution contains cross polarized terms in the scattered fields, which arise from a coupling of the $T E_{z}$ and $T M_{z}$ waves at the impedance boundary on the cylinder. Here, $z$ is the cylinder axis. Numerical results show very good accuracy for the simpler and efficient UTD solution, when compared to exact but very slowly convergent eigenfunction solution.
\end{abstract}

\section{INTRODUCTION}

A new UTD solution is developed for the canonical problem of the scattering of an obliquely incident EM plane wave by a circular cylinder with an impedance boundary condition (IBC). This UTD solution is uniformly valid across the surface shadow boundary (SSB), which is defined by an extension of incident wave at grazing on the cylinder surface. Furthermore, it reduces uniformly to the geometrical optics (GO) field in the deep lit region, and to the creeping wave surface diffracted field in the deep shadow region. It is important to note that this UTD solution exhibits a coupling of the $\mathrm{TE}_{\mathrm{z}}$ and $\mathrm{TM}_{\mathrm{z}}$ fields (with $\mathrm{z}$ being the cylinder axis) within the SSB transition region and the deep shadow region. As expected, the fields become decoupled in the deep lit region where GO is valid. Such a coupling of $\mathrm{TE}_{\mathrm{z}}$ and $\mathrm{TM}_{\mathrm{z}}$ fields vanishes for the perfectly conducting case, and also for the IBC case only when the plane wave is normally incident on the cylinder. This canonical UTD solution is crucial for the construction of a more general UTD solution valid for an arbitrary smooth convex surface with an IBC, when it is illuminated by an arbitrary EM ray optical field. An $e^{j \omega t}$ time convention is assumed and suppressed in the development of this UTD solution.

\section{A BRIEF SUMMARY OF TECHNICAL APPROACH}

The UTD solution for the above canonical problem is developed from an exact eigenfunction series solution, after the latter is converted from a series summation to a suitable integral form whose uniform asymptotic evaluation can be cast into a UTD ray format. The exact eigenfunction series expansion emphasizes waves propagating in the radial domain (with continuous eigenfunctions in the axial domain and discrete eigenfunctions in the circumferential domain); such an expansion is very slowly convergent for large cylinders. On the other hand, when the Watson transform is applied to this radially propagating eigenfunction series, it leads to an integral emphasizing circumferentially propagating waves which is suitable for treating electrically large cylinders. A uniform asymptotic evaluation of this latter integral is performed in a fashion similar to that presented previously in [1]. Others [2.3] have also followed the procedure in [1]; however, the solution in [2] is not uniform so that it is not valid everywhere around the cylinder, while that in [3] is valid only for the two dimensional cases of normal incidence on the cylinder with an IBC. The latter therefore does not exhibit cross polarization effects which can become significant for oblique angles of incidence on an IBC cylinder.

The exact eigenfunction series solution, which forms the basis of the UTD development here is in turn constructed from potential theory, where $\mathrm{TE}_{\mathrm{z}}$ and $\mathrm{TM}_{\mathrm{z}}$ potentials (along $\mathrm{z}$ ) are introduced to guarantee completeness. A uniform asymptotic evaluation of the integral for the $\mathrm{TE}_{z}$ and $\mathrm{TM}_{\mathrm{z}}$ fields resulting from the potentials, after their eigenfunction series representation is converted via the Watson transform into the required integral form, can subsequently be expressed in the ray coordinates. The UTD ray solution can then be identified immediately. 
The UTD solution is expressed in terms of what may be regarded as a generalization of Fock type integral transition functions, which keep the solution uniformly valid across the SSB, and reduces automatically to the non-uniform version that is valid in the deep lit and deep shadow regions exterior to the SSB transition region.

\section{CONCLUSION}

A UTD solution is developed for the canonical problem of the scattering of an obliquely incident plane wave which illuminates a circular cylinder with an IBC. The IBC can model a sufficiently thin material coating over a highly metallic surface, or a surface with an imperfectly conducting boundary condition. The numerical results based on this UTD solution show that it is accurate over a wide range of cylinder radius and surface impedance values provided the observer is not very close to the surface. This UTD solution also includes the effects of almost trapped surface waves (which leak energy due to surface curvature in this case). Also, this UTD solution properly includes cross polarization effects in the scattered fields arising from oblique incidence and IBC.

\section{References}

[1] P. H. Pathak, "An asymptotic analysis of the scattering of plane waves by a smooth convex cylinder," Radio Science, vol. 14, no.3, pp. 419435, May-Jun, 1979.

[2] H. T. Kim and N. Wang, "UTD solution for electromagnetic scattering by a circular cylinder with thin lossy coatings," IEEE Transactions on Antennas and Propagation, vol. 37, no.11, pp. 1463-1472, Nov, 1989.

[3] T. B. A. Senior and J. L. Volakis, "Approximate Boundary Conditions in Electromagnetics", IEE, London, UK, 1995. 